TAPROBANICA, ISSN 1800-427X. May, 2021. Vol. 10, No. 01: pp. 4-22, pls. 1-2.

(C) Research Center for Climate Change and Department of Biology, Faculty of Mathematics \& Natural Sciences, University of Indonesia, Depok 16424, INDONESIA.

http://www.taprobanica.org

https://doi.org/10.47605/tapro.v10i1.244

urn:lsid:zoobank.org:pub:D9E149DC-A345-4455-84B3-B8AF1A889451

\title{
A NEW SPECIES OF THE GENUS Tylototriton (AMPHIBIA, CAUDATA, SALAMANDRIDAE) FROM CENTRAL VIETNAM
}

\author{
Nikolay A. Poyarkov ${ }^{1,2}$, Tan Van Nguyen $^{3} \&$ Dmitriy V. Arkhipov ${ }^{1}$ \\ ${ }^{1}$ Department of Vertebrate Zoology, Biological Faculty, Lomonosov Moscow State University, Moscow, Russia \\ ${ }^{2}$ Joint Russian-Vietnamese Tropical Research and Technological Center, Hanoi, Vietnam \\ ${ }^{3}$ Department of Species Conservation, Save Vietnam's Wildlife, Ninh Binh, Vietnam \\ Corresponding author.E-mail: n.poyarkov@gmail.com ${ }^{2}$
}

\begin{abstract}
We describe a new species of the genus Tylototriton from $\mathrm{Pu}$ Hoat Nature Reserve, Nghe An Province, in the northern part of Central Vietnam, based on morphological and molecular evidence. The new species was previously confused with $T$. vietnamensis from northern Vietnam and $T$. notialis from Laos. The new species can be distinguished from $T$. notialis by the absence of orange coloration on posterior end of parotids ( $v s$ presence); an indistinct brown coloration on rib nodules ( $v s$ bright orange); a broader and slightly rounded head ( $v s$ narrower and angular); comparatively shorter limbs ( $v s$ longer); slightly fewer number, smaller size and irregular arrangement of rib nodules ( $v s$ larger rib nodules arranged in two dorsolateral series). Phylogenetic analysis of the ND2 and 16S rRNA mtDNA genes confirmed the placement of the new species to Clade I of the subgenus Yaotriton, and suggests it is a sister species of T. notialis ( $p$-distance $3.0 \%$ in ND2 gene). The range of the new species is restricted to the $\mathrm{Pu}$ Hoat Mountain Range and is isolated from the range of T. notialis, which inhabits Northern Annamites (Truong Son) Mountains by the valley of the Ca River, an important biogeographic barrier. The new species is currently known only from the montane forests of $\mathrm{Pu}$ Hoat Nature Reserve (at elevations from 700 to $1000 \mathrm{~m}$ a.s.l.). We suggest the new species be classified as Endangered (EN) on the IUCN Red List.
\end{abstract}

Key words: Tylototriton thaiorum sp. nov., mtDNA genealogy, Pu Hoat Nature Reserve, Ca River

\section{Introduction}

The salamandrid genus Tylototriton Anderson, 1871 (colloquially known as crocodile newts) currently includes 31 recognized species, inhabiting montane forest areas throughout the Asian monsoon climate zone from eastern Himalaya, southern and central China including
Hainan Island, to northern Indochina including northern Vietnam, Laos, northern Thailand, and Myanmar (Wang et al. 2018, Bernardes et al. 2020, Frost 2021). The genus is traditionally partitioned into two subgenera: Tylototriton sensu stricto is characterized by the presence of orange spots on the head, dorsum, tail, or sides 
of the body, while the subgenus Yaotriton is characterized by generally much darker almost blackish coloration (Yang et al. 2014, Li et al. 2020), though the validity of this subgeneric taxonomy has been questioned in a number of studies (e.g. Wang et al. 2018, Than Zaw et al. 2019). Several recent phylogenetic studies also demonstrated that our knowledge on taxonomic diversity of the genus Tylototriton is still far from complete, indicating the presence of numerous undescribed morphologically cryptic lineages, which actually might represent independent species (Wang et al. 2018, Than Zaw et al. 2019). The amount of undescribed diversity appears to be especially high in the $T$. asperrimus group of the subgenus Yaotriton (Bernardes et al. 2020, Li et al. 2020). The number of species of Tylototriton in Vietnam is high, with seven species of this genus having been recorded so far for the country, namely $T$. anguliceps Le, Nguyen, Nishikawa et al. 2015, T. notialis Stuart, Phimmachak, Sivongxay \& Robichaud, 2010, T. pasmansi Bernardes, Le, Nguyen et al., 2020, T. cf. pulcherrimus Hou, Zhang, Li \& Lu, 2012, T. sparreboomi Bernardes, Le, Nguyen et al., 2020, T. vietnamensis Böhme, Schöttler, Nguyen \& Köhler, 2005, and T. ziegleri Nishikawa, Matsui \& Nguyen, 2013a (Böhme et al. 2005, Nishikawa et al. 2013b, Le et al. 2015, Fei \& Ye 2016, Bernardes et al. 2020). It is noteworthy that six of the seven Tylototriton species recorded from Vietnam were described during the last ten years. Three other species, namely $T$. asperrimus Unterstein, 1930 (presently restricted to southeastern China), T. verrucosus Anderson, 1871 (presently restricted to Yunnan Province of China, northern Myanmar and Thailand), and $T$. shanjing Nussbaum, Brodie \& Yang, 1995 (presently restricted to Yunnan Province of China) also have been reported from Vietnam (Nguyen et al. 2009, Hernandez 2016). However, recent phylogenetic studies suggest that these records were likely based on misidentifications of $T$. pasmansi or $T$. sparreboomi, and $T$. anguliceps complex members, respectively (see Le et al. 2015, Bernardes et al. 2020).

Originally, the occurrence of Tylototriton in $\mathrm{Pu}$ Hoat Forest, Dong Van Commune, Que Phong District, Nghe An Province of Vietnam (Fig. 1, locality 1) was reported by Böhme et al. (2005) based on personal observations of $\mathrm{T}$. Schöttler. Böhme et al. (2005) provisionally assigned this population to $T$. vietnamensis, without providing any information on voucher specimens or any other justification for this taxonomy. Soon afterwards, Stuart et al. (2010) described a new species $T$. notialis based on three adult specimens from Nakai-Nam Theun National Protected Area, Khammouan Province of Laos (Fig. 1, locality 2). Nishikawa et al. (2013b) briefly mentioned the specimens from $\mathrm{Pu}$ Hoat and assigned them to $T$. notialis, providing further evidence for the full species status of this taxon as distinct from $T$. asperrimus. At the same time, Nishikawa et al. (2013b) noted differences in coloration between the true T. notialis from Laos, which has orange coloration on the posterior ends of the parotoid glands, and the $\mathrm{Pu}$ Hoat population, which lacks orange coloration on the parotoids. Nevertheless owing to small genetic distance between $T$. notialis sensu stricto and the $\mathrm{Pu}$ Hoat population, Nishikawa et al. (2013b) concluded that both populations likely belong to the same species. More recently Nishikawa et al. (2020) reported on a new locality of $T$. notialis sensu stricto in the close vicinity of the type locality of this species in Khammouan Province of Laos, and stated that "it seems that the only morphological difference between the populations [of this species] from Laos and Vietnam is the presence or absence of the colorful markings" (Nishikawa et al. 2020: 135). However, the limited number of specimens examined by Stuart et al. (2010) and Nishikawa et al. (2013b, 2020) leaves open the question as to the correct identification of morphological differences between these two populations.

In May 2019, during fieldwork in the montane forests of $\mathrm{Pu}$ Hoat Nature Reserve in Nghe An Province of Vietnam (Fig. 1, locality 1), we collected a series of six specimens of a newt, which was tentatively identified as Tylototriton sp. In the present study, based on this new material we reexamine and compare the morphological and molecular characters of the $\mathrm{Pu}$ Hoat Tylototriton population with $T$. notialis sensu stricto from Laos, and demonstrate that the concordant differences between them in mtDNA, external morphology and coloration require the recognition of the $\mathrm{Pu}$ Hoat population as a distinct species.

\section{Material and methods}

Sample collection: Fieldwork was carried out in $\mathrm{Pu}$ Hoat Nature Reserve in the environs of Muong Dan Village, Hanh Dich Commune, Que Phong District, Nghe An Province, northern part 
of Central Vietnam (Fig. 1, locality 1), from 0615 May 2018 and from 15-30 May 2019. Specimens of Tylototriton sp. were collected by hand in swamps in forest clearings surrounded by montane evergreen tropical forests. Geographic coordinates and altitude were obtained using a Garmin GPSMAP 60CSx GPS receiver (Garmin Ltd., USA) and recorded in datum WGS 84. Specimens were euthanized by $20 \%$ benzocaine and tissue samples (liver) for genetic analysis were taken and stored in $96 \%$ ethanol prior to preservation. Specimens were subsequently preserved in $70 \%$ ethanol and deposited in the herpetological collection of the Zoological Museum of Moscow State University (ZMMU) in Moscow, Russia.

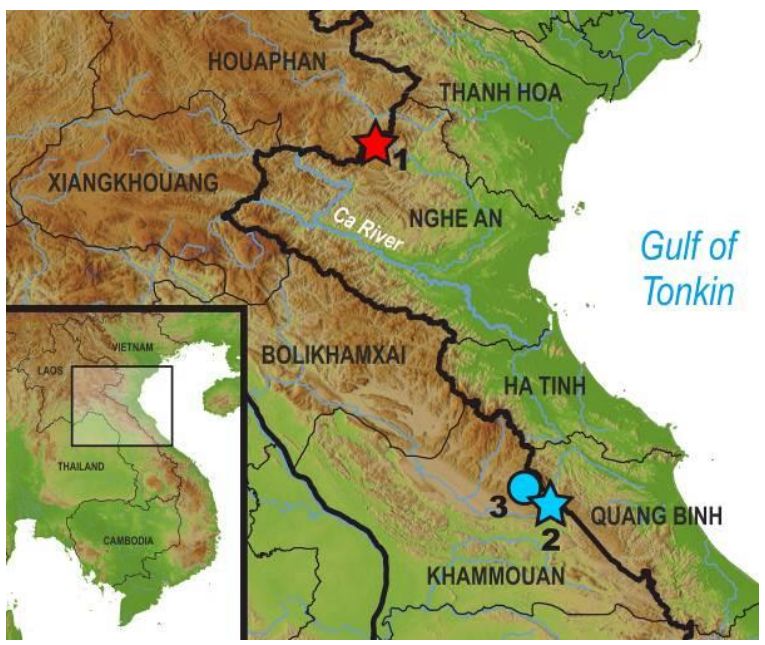

Figure 1. Distribution of Tylototriton notialis sensu stricto (blue) and the new species (red) in Central Vietnam and Laos; inset shows the location of this area on the map of Indochina. 1, Pu Hoat Nature Reserve, Nghe An Province, Vietnam; 2, Nam On River, Nakai-Nam Theun National Protected Area, Khammouan Province, Laos; 3, Mt. Jeung, Khammouan Province, Laos. Stars denote the type localities of $T$. notialis and the new species.

Morphological description: Specimens of Tylototriton sp. were photographed in life and after preservation. The sex and maturity of the specimens were checked by minor dissections. Measurements were taken using a digital caliper to the nearest $0.01 \mathrm{~mm}$, subsequently rounded to $0.1 \mathrm{~mm}$. We used a stereoscopic light binocular microscope when necessary. Morphometrics followed Okamiya et al. (2018) and Than Zaw et al. (2019) and included the following 24 measurements taken for the type series and used in inter-specific comparisons: (1) SVL (snoutvent length) from tip of snout to anterior tip of vent; (2) HL (head length); (3) HW (head width); (4) MXHW (maximum head width); (5) IND (internarial distance); (6) AGD (axillagroin distance); (7) TRL (trunk length); (8) TAL (tail length) from anterior tip of vent to tail tip; (9) VL (vent length); (10) FLL (forelimb length); (11) HLL (hindlimb length); (12) VTW (vomerine tooth series width): greatest width of vomerine tooth series; (13) VTL (vomerine tooth series length): greatest length of vomerine tooth series; (14) LJL (lower jaw length from tip of lower jaw to articulation of upper and lower jaws); (15) SL (snout length from tip of snout to anterior tip of upper eyelid); (16) IOD (minimum interorbital distance); (17) UEW (maximum upper eyelid width); (18) UEL (upper eyelid length, distance between anterior and posterior angles); (19) OL (orbit length); (20) BTAW (basal tail width at level of anterior tip of cloaca); (21) MTAW (tail width at midlevel of tail); (22) MXTAH (maximum tail height); (23) MTAH (tail height at mid-level of tail); and (24) ON (orbitonarial distance). For holotype description, we additionally examined the following 12 morphometric characters following Poyarkov et al. (2012) and Than Zaw et al. 2019: (25) ICD (intercanthal distance); (26) CW (chest width); (27) NSD (nostril-snout distance); (28) 1FL (first finger length from base to tip); (29) 2FL (second finger length from base to tip); (30) 3FL (third finger length from base to tip); (31) 4FL (fourth finger length from base to tip); (32) 1TL (first toe length from base to tip); (33) 2TL (second toe length from base to tip); (34) 3TL (third toe length from base to tip); (35) 4TL (fourth toe length from base to tip); (36) 5TL (fifth toe length from base to tip).

The diagnosis of the subgenus Yaotriton and morphological characters for comparison were taken from original descriptions and taxonomic reviews of the genus: Bernardes et al. (2020), Böhme et al. (2005), Chen et al. (2010), Fei \& Ye (2016), Hou et al. (2012), Li et al. (2020), Nishikawa et al. (2013a, 2013b, 2020), Phimmachak et al. (2015), Qian et al. (2017), Shen et al. (2012), Stuart et al. (2010), Than Zaw et al. (2019), and Yang et al. (2014).

Laboratory methods: Total genomic DNA was extracted from 95\% ethanol-preserved muscle tissues using standard phenol-chloroform extraction protocols (Hillis et al. 1996). Total DNA concentration was estimated in $1 \mu \mathrm{L}$ using a NanoDrop 2000 spectrophotometer (Thermo Scientific, USA), and consequently adjusted to 100 ng DNA $/ \mu \mathrm{L}$. We amplified two mtDNA fragments including the partial sequences of the 
ND2 and 16S rRNA mtDNA genes. These markers were chosen as they were proven to be useful in studies of Tylototriton phylogeny and taxonomy (Nishikawa et al. 2013a, 2013b, 2014, Wang et al. 2018, Than Zaw et al. 2019, and references therein). We used the $16 \mathrm{~L}-1$ (forward) (5'-CTGACCGTGCAAA GGTAGCG TAATCACT-3') and 16H-1 (reverse) (5'-CTCCG GTCTGAACTCAGATCACGTAGG-3') primers to amplify the $16 \mathrm{~S}$ rRNA fragments following Hedges (1994). For amplification and sequencing of the ND2 gene, we used the SL-1 (forward) (5'-ATAGAGGTTCAAACCCTCTC-3') and SL-2 (reverse) (5'- TTAAAGTGTCTGGGTT GCATTCAG-3') primers of Wang et al. (2018). Polymerase chain reaction (PCR) conditions followed Than Zaw et al. (2019). PCR was performed in $20 \mu \mathrm{L}$ using $50 \mathrm{ng}$ genomic DNA, $10 \mathrm{nmol}$ of each primer, $15 \mathrm{nmol}$ of each dNTP, $50 \mathrm{nmol}$ additional $\mathrm{MgCl} 2$, Taq PCR buffer (10 $\mathrm{mmol} / \mathrm{L}$ Tris- $\mathrm{HCl}, \mathrm{pH} 8.3,50 \mathrm{mmol} / \mathrm{L} \mathrm{KCl}, 1.1$ $\mathrm{mmol} / \mathrm{L} \mathrm{MgCl} 2$, and $0.01 \%$ gelatin), and $1 \mathrm{U}$ of Taq DNA polymerase. PCR cycles included an initial denaturation step of $4 \mathrm{~min}$ at $94^{\circ} \mathrm{C}$ and 35 cycles of denaturation for $30 \mathrm{~s}$ at $94{ }^{\circ} \mathrm{C}$, primer annealing for $30 \mathrm{~s}$ at $48-58{ }^{\circ} \mathrm{C}$, and extension for $1 \mathrm{~min} 30 \mathrm{~s}$ at $72{ }^{\circ} \mathrm{C}$. PCR products were visualized by agarose gel electrophoresis in the presence of ethidium bromide and consequently purified using $2 \mu \mathrm{L}$ from a 1: 4 dilution of ExoSapIt (Amersham, UK) per $5 \mu \mathrm{L}$ of PCR product prior to cycle sequencing. Sequencing was performed in both directions using the same primers as used in PCR on an ABI3730xl automated sequencer (Applied Biosystems, USA) at Evrogen Inc., Moscow (Russia). The newly obtained sequences were aligned and deposited in GenBank under the accession numbers MW883478-MW883485 (Table 1). Sequences of 31 other Tylototriton species used for comparisons along with three sequences of outgroup taxa were obtained from GenBank (see Table 1).

Phylogenetic analyses: Sequences of partial fragments of ND2 and 16S rRNA mtDNA for 51 Salamandridae specimens, including 48 representatives of Tylototriton (36 taxa) and three sequences of outgroup members of Salamandridae [Echinotriton andersoni (Boulenger, 1892), E. chinhaiensis (Chang, 1932), and Pleurodeles walt Michahelles, 1830] were included in the final alignment with a total length of up to $1665 \mathrm{bp}$. Information on voucher specimens and GenBank accession numbers used in phylogenetic analyses is summarized in
Table 1. Nucleotide sequences were initially aligned in MAFFT v.6 (Katoh et al. 2002) with default parameters, and then checked by eye and slightly adjusted in BioEdit 7.0.5.2 (Hall 1999). The dataset was divided into four partitions: three codon partitions for the ND2 gene and a single partition for 16S rRNA, with the optimal evolutionary models for each estimated using MODELTEST v. 3.06 (Posada \& Crandall 1998). According to the Akaike information criterion (AIC), for the ND2 gene the $\mathrm{HKY}+\mathrm{G}$ model was selected as the best fit for the first and second codon partitions, and the $\mathrm{J} 2+\mathrm{G}$ model was considered the best fit for the third codon partition; while the TVM+G model was the best fit for the 16S rRNA partition. Mean uncorrected genetic distances ( $p$-distances) between sequences were calculated in MEGA 7.0 with missing data and gaps excluded from the analysis (Kumar et al. 2016). The matrilineal genealogy was inferred using Bayesian inference (BI) and maximum likelihood (ML) algorithms. BI analyses were conducted in MrBayes v3.1.2 (Huelsenbeck \& Ronquist 2001, Ronquist \& Huelsenbeck 2003). Metropolis coupled Markov chain Monte Carlo (MCMCMC) analyses were run with one cold chain and three heated chains for twenty million generations and sampled every 2000 generations. Five independent MCMCMC runs were performed, the MCMC simulations ran for 100 million generations, were sampled every 10,000 generations, and the first $10 \%$ of the trees from each run from each data set were discarded as burn-in. We checked the convergence of the runs and that the effective sample sizes (ESS) were all above 200 by exploring the likelihood plots using TRACER v1.6 (Rambaut et al. 2014). Confidence in tree topology was tested by posterior probability (BI PP) for the BI trees (Huelsenbeck \& Ronquist 2001). Nodes with PP values over 0.95 were a-priori regarded as sufficiently resolved, those between 0.95 and 0.90 were regarded as tendencies, and values below 0.90 were considered to not be supported. We conducted ML analysis in the IQ-TREE webserver. One-thousand bootstrap pseudoreplicates via the ultrafast bootstrap (UFBS; Hoang et al. 2018) approximation algorithm were employed and nodes having ML UFBS values of 95 and above were a-priori considered highly supported, while the nodes with values of 90-94 were considered wellsupported, and the nodes with values of 70-89 were considered as tendencies (Bui et al. 2013). 
Table 1. Sequences and voucher specimens of Tylototriton and outgroup taxa (Echinotriton chinhaiensis, E. andersoni, Pleurodeles waltl) used in this study; L, lineage; NP, National Park; NR, Nature Reserve; Co., County; Dist., District; Mt. Mountain; H, holotype; — not available.

\begin{tabular}{|c|c|c|c|c|}
\hline Species name & Voucher Number & Locality & 16S rRNA & ND2 \\
\hline \multicolumn{5}{|l|}{ Ingroup: } \\
\hline T. anguliceps & TBU PAE671 & Copia NR, Thuan Chau, Son La, Vietnam & - & LC017833 \\
\hline T. anhuiensis & CIB 08042905-2 & Yuexi Co., Anhui, China & KY800587 & KY800853 \\
\hline T. asperrimus & CIB 70063 & Longsheng Co., Guangxi, China & KY800549 & KC147816 \\
\hline T. broadoridgus & CIB 200084 & Sangzhi Co., Hunan, China & KY800570 & KY800837 \\
\hline T. dabienicus & HNNU 1004-015 & Shangcheng Co., Anhui, China & KY800607 & KC147811 \\
\hline T. hainanensis & CIB 20081048 & Mt. Diaoluo, Hainan, China & KY800553 & KC147817 \\
\hline T. himalayanus & CIB 201406246 & Mai Pokhari, Illam, Mechi, Nepal & KY800590 & KT765173 \\
\hline T. kachinorum & ZMMU A5953 & In Gyi Taung Mt., Kachin, Myanmar & MK095618 & MK097273 \\
\hline T. kweichowensis & CIBW g20080818014 & Bijie City, Guizhou, China & KY800551 & KY800823 \\
\hline T. liuyangensis & CSUFT20100108 & Liuyang City, Hunan, China & KY800606 & KJ205598 \\
\hline T. lizhenchangi & KUHE 42316 & Yizhang Co., Hunan, China & KY800621 & KY800881 \\
\hline T. maolanensis & CIBW g 20090730001 & Libo Co., Guizhou, China & KY800575 & KY800842 \\
\hline T. ngarsuensis & LSUHC 13763 & Ngar Su, Taunggyi Dist., Shan, Myanmar & - & MH836585 \\
\hline T. notialis & FMNH HERP 271120 & Boualapha Dist., Khammouan, Laos & - & HM462061 \\
\hline T. notialis & FMNH HERP 271121 & Boualapha Dist., Khammouan, Laos & - & HM462062 \\
\hline T. notialis & FMNH HERP 271122 & Boualapha Dist., Khammouan, Laos & - & HM462063 \\
\hline T. notialis & FES RA.18.001 & Mt. Jeung, Boualapha, Khammouan, Laos & - & LC522466 \\
\hline T. panhai & PH019 & Phu Hin Rong Kla NP, Thailand & - & AB830735 \\
\hline T.panwaensis & CAS 245418 & Panwa, Myitkyina Dist., Kachin, Myanmar & - & KT304279 \\
\hline T. pasmansi obsti & IEBR 4471 & Xuan Nha NR, Van Ho, Son La, Vietnam & - & MT210168 \\
\hline T.p.pasmansi & IEBR 4466 & Phu Canh NR, Da Bac, Hoa Binh, Vietnam & - & MT201166 \\
\hline T. phukhaensis & CUMZ A-7717 & Doi Phu Kha NP, Nan, Thailand & - & MN912573 \\
\hline T. podichthys & IEBR A2014-1 & Xam Neua, Huaphanh, Laos & - & LC017835 \\
\hline T.pseudoverrucosus & CIB WCG2012012 & Ningnan Co., Liangshanyizu, China & KY800599 & KY800860 \\
\hline T. pulcherrimus & CIB TY040 & Lüchun Co., Yunnan, China & KY800626 & KY800890 \\
\hline T. shanjing & KIZ 201306081 & Yongde Co., Yunnan, China & KY800593 & KY800856 \\
\hline T. shanorum & CAS 230940 & Taunggyi Township, Shan, Myanmar & - & AB922823 \\
\hline T. sparreboomi & IEBR 4476 & Sin Ho, Lai Chau, Vietnam & - & MT210162 \\
\hline T. taliangensis & CIB GG200110183 & Shimian Co., Yan'an City, Sichuan, China & KY800559 & KC147819 \\
\hline T. thaiorum sp. nov. & ZMMU A- $7577^{\mathrm{H}}$ & Pu Hoat NR, Nghe An, Vietnam & MW883482 & MW883478 \\
\hline T. thaiorum sp. nov. & ZMMU A-7578 & Pu Hoat NR, Nghe An, Vietnam & MW883483 & MW883479 \\
\hline T. thaiorum sp. nov. & ZMMU A-7579 & Pu Hoat NR, Nghe An, Vietnam & MW883484 & MW883480 \\
\hline T. thaiorum sp. nov. & ZMMU A-7580 & Pu Hoat NR, Nghe An, Vietnam & MW883485 & MW883481 \\
\hline T. thaiorum sp. nov. & VNMN TAO1229 & Pu Hoat NR, Nghe An, Vietnam & - & KY800883 \\
\hline T. thaiorum sp. nov. & VNMN TAO1235 & Pu Hoat NR, Nghe An, Vietnam & - & KY800884 \\
\hline T. uyenoi & KUHE 19037 & Doi Inthanon, Chiang Mai, Thailand & - & AB830730 \\
\hline T. verrucosus & CIB TSHS1 & Longchuan Co., Dehong, Yunnan, China & KY800581 & KY800847 \\
\hline T. vietnamensis & IEBR A.3674 & Tay Yen Tu NR, Bac Giang, Vietnam & KY800614 & KY800874 \\
\hline T. wenxianensis $\mathrm{L} 1$ & CIB 20090527 & Wenxian Co., Gansu, China & KY800579 & KC147813 \\
\hline T. wenxianensis $\mathrm{L} 2$ & CIB 20070638 & Qingchuan Co., Sichuan, China & KY800543 & KY800816 \\
\hline T. wenxianensis L3 & CIB 20090601 & Wangcang Co., Sichuan, China & KY800571 & KY800838 \\
\hline T. wenxianensis $\mathrm{L} 4$ & CIB 20080002 & Yunyang Co., Chongqing, China & KY800540 & KY800813 \\
\hline T. wenxianensis L5 & CIB 20080003 & Yunyang Co., Chongqing, China & KY800541 & KY800814 \\
\hline T. yangi & KUHE 42282 & Pingbian Co., Yunnan, China & KY800624 & KY800887 \\
\hline T. ziegleri & VNMN 3390 & Quan Ba, Ha Giang, Vietnam & KY800625 & KY800889 \\
\hline Tylototriton sp. 1 & CIB WG200600019 & Suiyang Co., Zunyi, Guizhou, China & KY800544 & KY800817 \\
\hline Tylototriton sp. 2 & CIB WH10001 & Wufeng Co., Hubei, China & KY800600 & KY800863 \\
\hline Tylototriton sp. 3 & CIB XZ20091201 & Xinyi City, Guangdong, China & KY800616 & KY800876 \\
\hline \multicolumn{5}{|l|}{ Outgroup: } \\
\hline E. chinhaiensis & CIB ZHJY1 & Zhenhai Co., Zhejiang, China & KY800627 & KY800891 \\
\hline E. andersoni & MVZ 232187 & Tokunoshima, Kagoshima, Japan & EU880314 & EU880314 \\
\hline P.waltl & MVZ 231894 & Cadiz, Andalusia, Spain & EU880330 & EU880330 \\
\hline
\end{tabular}




\section{Results}

Sequence variation. The final alignment of the ND2 gene contained 1067 aligned nucleotides, of which, 645 sites were conserved and 422 sites were variable, of which 276 were parsimonyinformative. The transition-transversion bias ( $\mathrm{R})$ was estimated as 5.17. Nucleotide frequencies were $37.54 \%$ (A), $23.81 \%$ (T), $28.23 \%$ (C), and $10.42 \%(\mathrm{G})$. The final alignment of the $16 \mathrm{~S}$ rRNA gene contained 508 aligned characters, of which, 424 sites were conserved and 82 sites were variable, of which 53 were suggested as parsimony-informative. The transitiontransversion bias (R) was estimated as 7.45. Nucleotide frequencies were $36.97 \%$ (A), $24.81 \%$ (T), $20.29 \%$ (C), and $17.93 \%$ (G) (all data given for ingroup only).

MtDNA genealogy: $\mathrm{BI}$ and ML phylogenetic analyses resulted in almost identical topologies (Fig. 2). The topology of the matrilineal genealogies was largely consistent with the phylogeny of Tylototriton presented by Wang et al. (2018) and Than Zaw et al. (2019) and recovered five main clades grouped into two major reciprocally monophyletic groups, corresponding to the subgenera Tylototriton sensu stricto and Yaotriton (Fig. 2). Monophyly of the subgenus Yaotriton was well-supported by ML analysis, and got no significant support in BI analysis (Fig. 2, 91/0.78, hereafter node values given for UFBS/BI PP, respectively); this group included three highly supported main clades:

(1) Clade 1 includes the members of the Tylototriton asperrimus species group from China, northern Vietnam and Laos, and included six nominal Tylototriton species: $T$. asperrimus, T. hainanensis Fei, Ye \& Yang, 1984, T. notialis, T. pasmansi (with two subspecies $T$. pasmansi pasmansi and $T$. pasmansi obsti), T. sparreboomi, and $T$. ziegleri, as well as one undescribed lineage Tylototriton sp. 3 from Guangdong Province of China previously identified as $T$. asperrimus (see Wang et al. 2018), and the population of Tylototriton sp. from Pu Hoat Nature Reserve.

(2) Clade 2 joined the members of the Tylototriton wenxianensis species group from China, and included seven species of Tylototriton: T. anhuiensis Qian, Sun, Li et al., 2017, T. broadoridgus Shen, Jiang \& Mo, 2012, T. dabienicus Chen, Wang \& Tao, 2010, T. liuyangensis Yang, Jiang, Shen \&
Fei, 2014, T. lizhenchangi Hou, Zhang, Jiang et al., 2012, T. maolanensis Li, Wei, Cheng et al., 2020, and T. wenxianensis Fei, Ye \& Yang, 1984, as well as two undescribed lineages Tylototriton sp. 1 from Guizhou and Tylototriton sp. 2 from Hubei provinces of China.

(3) Clade 3, which we refer to here as the $T$. vietnamensis species group, included two peculiar species of Tylototriton from northern Vietnam and north-eastern Thailand and adjacent Laos: T. panhai Nishikawa, Khonsue, Pomchote \& Matsui, 2013 and T. vietnamensis.

Monophyly of the subgenus Tylototriton sensu stricto received strong support both in ML and BI analyses (Fig. 2, 98/1.0); this group included two strongly supported clades:

(4) Clade 4 joined the members of the $T$. verrucosus species group from Nepal, Myanmar, China and northern Indochina, and included 14 nominal species of Tylototriton: T. anguliceps, T. himalayanus Khatiwada, Wang, Ghimire et al., 2015, T. kachinorum Zaw, Lay, Pawangkhanant et al., 2019, T. kweichowensis Fang \& Chang, 1932, T. ngarsuensis Grismer, Wood, Quah et al., 2018, T. panwaensis Grismer, Wood, Quah et al., 2019, T. phukaensis Pomchote, Khonsue, Thammachoti et al., 2020, T. podichthys Phimmachak, Aowphol \& Stuart, 2015, T. pulcherrimus, T. shanjing, $T$. shanorum Nishikawa, Matsui \& Rao, 2014, T. uyenoi Nishikawa, Khonsue, Pomchote \& Matsui, 2013, T. verrucosus, and T. yangi Hou, Zhang, Zhou et al., 2012.

(5) Clade 5, which we refer to here as the $T$. taliangensis species group, included two species from China, namely $T$. pseudoverrucosus $\mathrm{Hou}, \mathrm{Gu}$, Zhang et al., 2012 and T. taliangensis Liu, 1950.

Following the results of earlier studies (Wang et al. 2018, Than Zaw et al. 2019, Bernardes et al. 2020, Li et al. 2020), our analysis indicated the presence of several divergent lineages of Tylototriton, which might correspond to species that are yet to be described, referred to here as Tylototriton spp. 1-3 (see Table 1, Fig. 2). All these lineages belong to the subgenus Yaotriton and were until recently confused with either $T$. asperrimus or $T$. 
wenxianensis. Our analysis also indicated deep phylogenetic structuring within $T$. wenxianensis which consists of five mtDNA lineages, forming a well-supported clade (Fig. 2, 96/0.98). Overall, these results suggest that the taxonomy of this group remains incomplete and further taxonomic and phylogenetic research is needed.

The population of Tylototriton sp. from $\mathrm{Pu}$ Hoat belongs to clade 1 (Fig. 2), where it is reconstructed as a sister lineage of $T$. notialis from Laos with high levels of node support (99/1.0). The group formed by these two species is suggested to be a sister clade of the group including the members of the $T$. asperrimus species complex from Guangxi Province of China and northern Vietnam (T. asperrimus, $T$. pasmansi, and $T$. sparreboomi), although the phylogenetic position of the latter species is poorly supported; 85/0.60).

Genetic distances: The interspecific uncorrected genetic $p$-distances between the sequences of the ND2 mtDNA gene of Tylototriton sp. from $\mathrm{Pu}$ Hoat and other congeners varied from $3.0 \%$ (between Tylototriton sp. and its sister species T. notialis) to $13.5 \%$ (between Tylototriton sp. and $T$. ngarsuensis) (see Table 2). There was no intraspecific variation in ND2 gene sequences for Tylototriton sp. from Pu Hoat; for T. notialis it comprised $0.2 \%$ of substitutions (Table 2). The mtDNA lineages within $T$. wenxianensis were moderately divergent in ND2 gene sequences ( $p=2.3 \%$, Table 2$)$.

Systematics. Our phylogenetic analysis has unambiguously placed the Pu Hoat population of Tylototriton $\mathrm{sp}$. as a sister lineage of T. notialis, in agreement with earlier phylogenies of the group (Wang et al. 2018, Than Zaw et al. 2019). Additionally, the degree of pairwise divergence in the ND2 mtDNA gene of this population is comparable or greater than the level of genetic divergence observed between many recognized species of Tylototriton, within both the subgenus Tylototriton $[T$. verrucosus and $T$. shanjing (1.5\%), T. verrucosus and $T$. pulcherrimus (2.6\%), T. verrucosus and T. panwaensis (2.2\%), $T$. pulcherrimus and $T$. panwaensis (1.8\%), $T$. taliangensis and T. pseudoverrucosus (2.4\%), T. ngarsuensis and $T$. shanorum $(0.5 \%), T$. panwaensis and $T$. podichthys (2.9\%)] and the subgenus Yaotriton $[T$. anhuiensis and $T$. broadoridgus (2.6\%), T. anhuiensis and $T$. maolanensis $(2.6 \%), \quad T$. dabienicus and Tylototriton sp. 2 (2.6\%), T. anhuiensis and Tylototriton sp. 2 (2.6\%), T. broadoridgus and
Tylototriton sp. 2 (2.2\%)] (see Table 2). This and the phylogeny raise the question as to whether the Pu Hoat population of Tylototriton sp. is conspecific with $T$. notialis, as was suggested earlier (Nishikawa et al. 2013b, 2020), or whether it warrants taxonomic recognition as an independent taxon, which an integrative taxonomic approach might reveal?

Earlier works have reported morphological data only for a single male specimen of Tylototriton sp. from $\mathrm{Pu}$ Hoat Nature Reserve (Nishikawa et al. 2013b). Due to this limited sampling, only characters of body coloration were assessed. Nishikawa et al. (2013b) noted significant differences in coloration between the $\mathrm{Pu}$ Hoat Tylototriton sp. and T. notialis but interpreted these differences as intraspecific variation and concluded that "the presence of orange coloration on the parotoid cannot be used as a diagnostic characteristic for $T$. notialis" (Nishikawa et al. 2013b: 46). Recently, examining newly collected material on $T$. notialis from Laos, Nishikawa et al. (2020) argued that the only morphological difference between the populations from Laos and Vietnam is the presence or absence of the colorful markings (Nishikawa et al. 2020: 136). However, a detailed morphological examination of the $\mathrm{Pu}$ Hoat population of Tylototriton was never completed.

Here, based on newly collected material of Tylototriton sp. from Pu Hoat, we examine the external morphology and coloration of this population, and report on a number of diagnostically important characters readily distinguishing this population from $T$. notialis and all other congeners. These include not only the coloration of parotids and rib nodules, but also the shape and width of the head, relative length of limbs, size and arrangement of rib nodules. Moreover, the presently known ranges of T. notialis and the $\mathrm{Pu}$ Hoat populations are separated by the $\mathrm{Ca}$ River valley, which is considered an important biogeographic barrier, separating the subtropical montane forests of northern Vietnam from the tropical montane forests of the Northern Annamites (Truong Son Range) (Abramov \& Tran 2017; Poyarkov et al. 2021 in press). These arguments together support our hypothesis that the $\mathrm{Pu}$ Hoat population of Tylototriton represents a previously unknown species, which we describe below. 


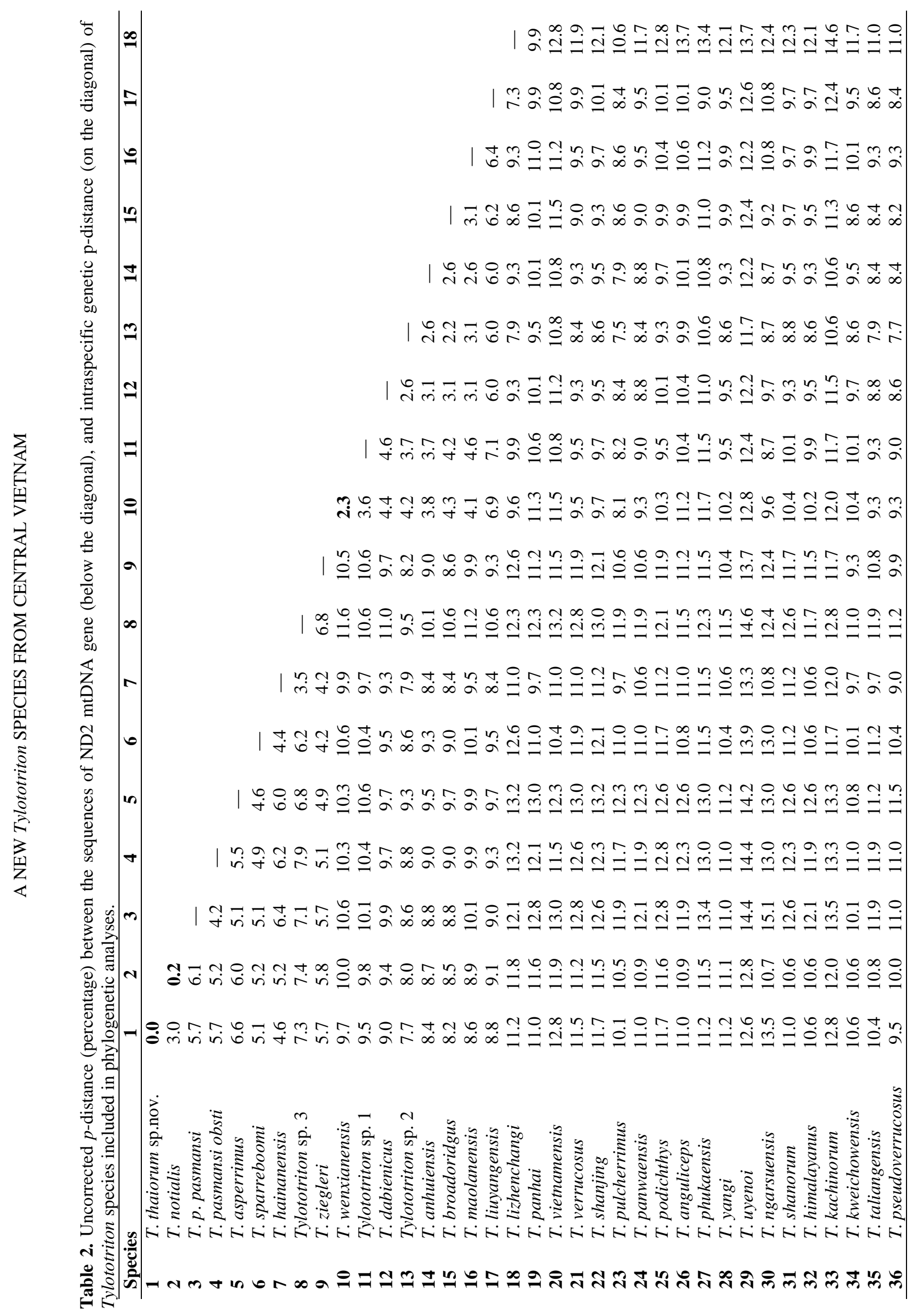




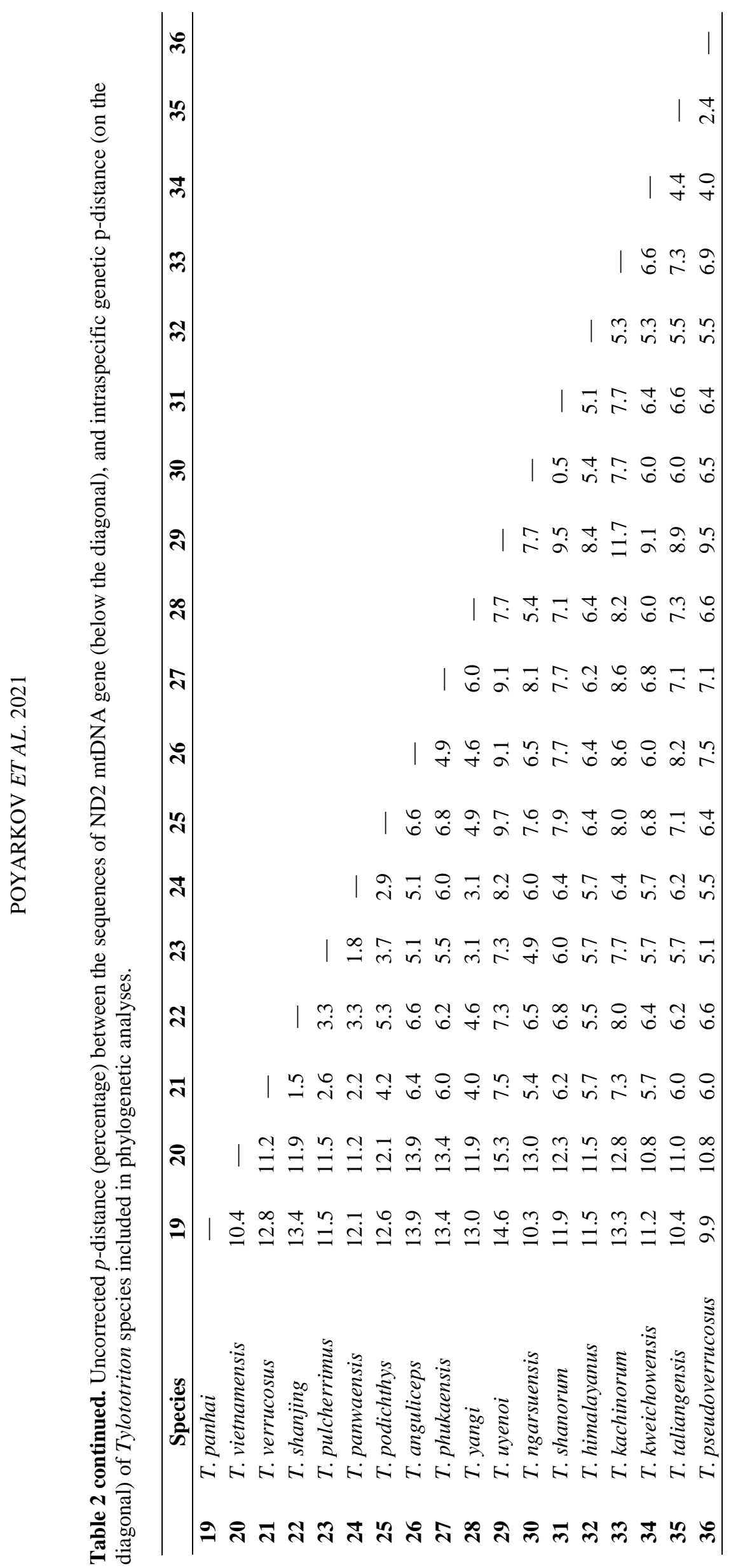




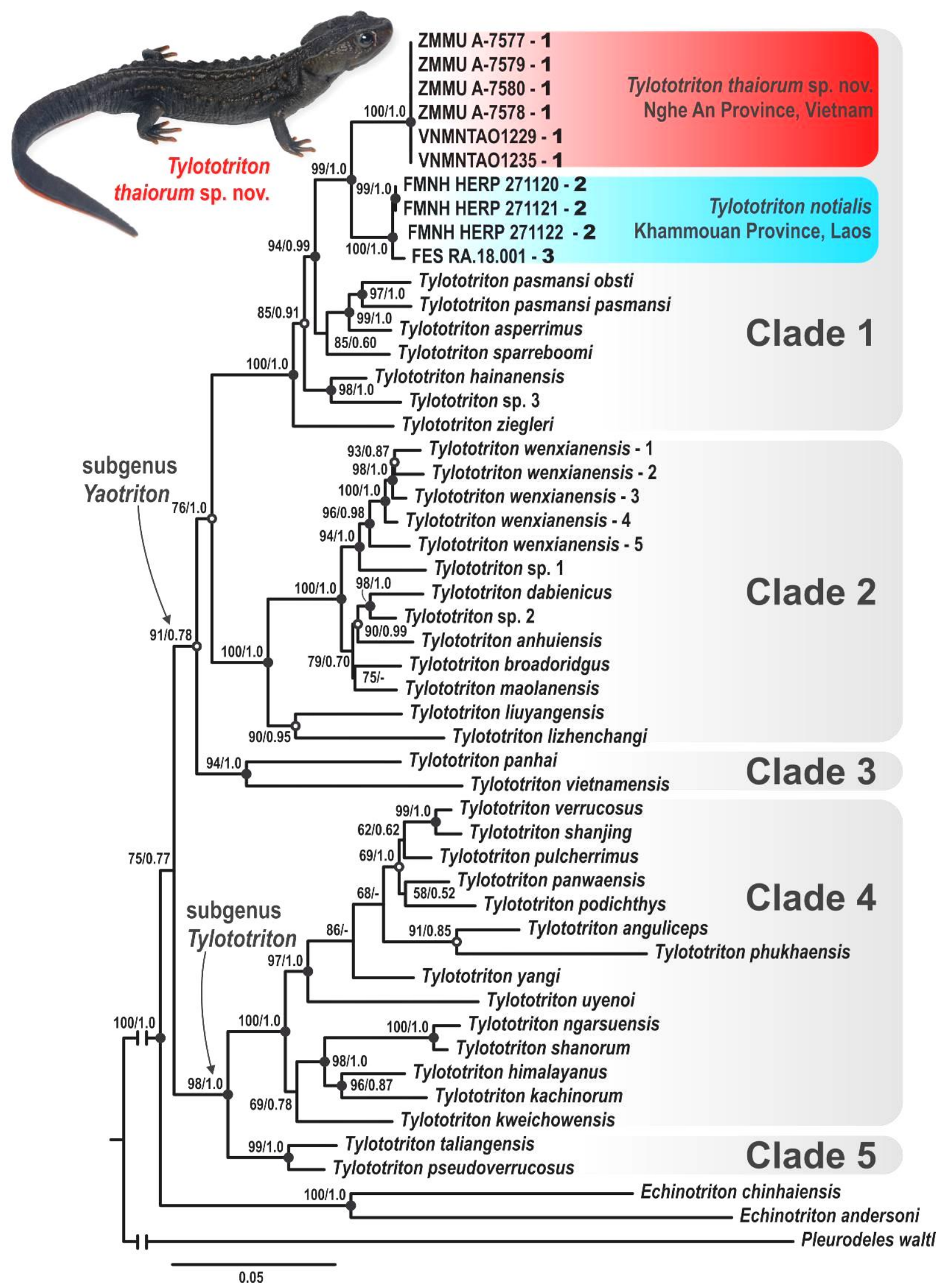

Figure 2. Maximum Likelihood tree of Tylototriton derived from the analysis of $1665 \mathrm{bp}$ of alignment including ND2 and 16S rRNA gene fragments. For voucher specimen information and GenBank accession numbers see Table 1. Red and blue colours denote the new species and T. notialis sensu stricto, respectively; numbers in bold after the specimen IDs correspond to the locality numbers in Fig. 1. Numbers at tree nodes correspond to UFBS/PP support values, respectively; well-supported nodes are marked with solid circle, moderate-support is indicated with empty circle, no circle means no-support. (C) Photo: Nikolay A. Poyarkov. 
Salamandridae Goldfuss, 1820

Tylototriton Anderson, 1871

Tylototriton thaiorum sp. nov.

[urn:1sid:zoobank.org:act:D7E7A23D-72FE-416E-BFE71D14B03612FF]

(Figs. 3-5; Tables 3-4)

Tylototriton vietnamensis — Böhme et al. 2005 [partim]

Tylototriton notialis - Nishikawa et al. 2013b [partim], Phimmachak et al. 2015 [partim], Yang et al. 2018 [partim], Than Zaw et al. 2019 [partim], Bernardes et al. 2020 [partim]

Holotype. Adult male, ZMMU A-7577 (field number NAP-08957), collected from an ephemeral swamp in a forest clearing surrounded by montane evergreen tropical forest in $\mathrm{Pu}$ Hoat Nature Reserve, environs of Muong Dan Village, Hanh Dich Commune, Que Phong District, Nghe An Province, Central Vietnam $\left(19.71715^{\circ} \mathrm{N}, 104.83791^{\circ} \mathrm{E} ; 709 \mathrm{~m}\right.$ a.s.l.), by Nikolay A. Poyarkov on 20th of May, 2019.

Paratypes $(\boldsymbol{n}=5)$. All adult males [ZMMU A-7578 (field number NAP-08958), and ZMMU A-7579-7682 (field numbers NAP-0904909052)], all from the same locality and with the same collection information as the holotype.

Diagnosis. The new species is assigned to the genus Tylototriton and the subgenus Yaotriton based on the results of the molecular phylogenetic analyses and the following combination of morphological attributes: presence of dorsal granules, dorsolateral bony ridges on head, presence of dorsolateral series of knob-like warts (rib nodules), and absence of quadrate spine (Figs. 3-5). Tylototriton thaiorum sp. nov. is differentiated from all other members of the subgenus Yaotrion by the following combination of morphological characters: medium body size (SVL 59.3-68.6 $\mathrm{mm}$ in males); snout obtusely rounded in dorsal view and in lateral profile; skin rough with fine granules; supratemporal bony ridges on head wide, slightly protruding, beginning at the anterior corner of orbit; sagittal ridge on head low and almost indistinct; limbs thin, tips of forelimb and hindlimb overlapping when adpressed along body; vertebral ridge distinct, rough, not segmented; rib nodules 10-14, small, poorly distinct, arranged in two irregular dorsolateral series intermittent with smaller warts; dorsal background coloration dark-brown, ventral coloration greyish brown, limbs blackish; fingers and toe tips, parts of soles and palms, and vent bright orange, orange stripe continuing along the ventral edge of tail to the tail tip.

Description of holotype. A medium-sized male specimen in a good state of preservation. Head longer than wide (HW/HL 0.94), head notably wider than body; rounded hexagonal in shape in dorsal view, depressed, gently sloping in profile; snout comparatively long, three times longer than eye (UEW/SL 0.38), obtusely rounded in dorsal view, rounded in lateral profile, projecting beyond lower jaw; nostrils on anterior margin of snout, located notably closer to snout tip than to eye (NSD/ON 0.61), with antero-lateral orientation, not visible from dorsal view; labial folds absent; tongue oval, attached to anterior floor of mouth, free posteriorly and laterally; vomerine tooth series in an inverted Vshape, converging anteriorly but not reaching choanae; parotoids distinct, large, crescentshaped, slightly projecting posteriorly; dorsolateral supratemporal bony ridges on head wide, slightly protruding, beginning at the anterior corner of orbit continuing to anterior end of parotoid, posterior ends slightly curved inside; sagittal bony ridge on head very weak, almost indistinct; gular fold present.

Body shape moderately stout; costal folds absent; vertebral middorsal ridge distinct, wide, not segmented, rough, running from occiput region to sacrum and anterior part of tail. Rib nodules poorly distinct, small, forming knoblike glandular warts, arranged in two irregular longitudinal series on dorsolateral surfaces of dorsum, 12 nodules placed on each side of body from posterior to axilla to level of posterior vent margin (base of tail). Size of rib nodules varies from rounded, distinctly knob-like anteriorly, to smaller and of more irregularly shaped posteriorly; rib nodules intermittent with smaller irregular warts, making dorsolateral series slightly ragged in shape.

Limbs comparatively thin; forelimbs slightly shorter than hindlimbs; relative lengths of limbs: FLL/SVL 0.31, HLL/SVL 0.33; fore- and hindlimbs overlapping when adpressed towards each other along body; fingers and toes well developed, free of webbing or folds; fingers four, comparative finger lengths: $1 \mathrm{FL}<4 \mathrm{FL}<2 \mathrm{FL}=3 \mathrm{FL}$; toes five, comparative toe lengths: $1 \mathrm{TL}=5 \mathrm{TL}<2 \mathrm{TL}<3 \mathrm{TL}=4 \mathrm{TL}$. Tail long, notably exceeding body length (TAL/SVL 1.12); laterally compressed along entire length, tapering posteriorly, lateral grooves on tail indistinct; dorsal tail fin starting at anterior one fifth of tail length, becoming higher and more 
distinct posteriorly, with maximal tail height at the middle of tail length, dorsal tail fin slightly serrated posteriorly; ventral tail ridge smooth; tail tip pointed.

Dorsal skin very rough, small granules and larger warts present on dorsal surfaces of head and dorsum, lateral sides of body and tail; ventral surfaces smoother with smaller granules arranged in transverse striations; throat with numerous tiny flat tubercles; surfaces of head ridges and middorsal vertebral ridge rough; limbs dorsally with numerous tiny tubercles, volar and plantar surfaces of hands and feet with tiny grooves forming reticulated pattern; flattened outer metacarpal and metatarsal tubercles distinct on palms and soles, respectively. Cloacal region slightly swollen, vent as a longitudinal slit, vent edges with numerous small transverse folds.

Colouration. In life, dorsum uniformly dark brown (Fig. 3); venter greyish brown, slightly lighter than dorsum; limbs blackish; rib nodules and vertebral ridge brown; fingers and toes bright reddish orange (ventrally along entire lengths, dorsally only on tips of the digits), parts of soles and palms corresponding to outer metatarsal and metacarpal tubercles reddishorange, vent and ventral tail ridge bright orange. After two years in preservative, dorsal coloration slightly faded, becoming dark grey, while orange markings faded to cream.

Measurements of holotype. (in mm). SVL 65.2; HL 15.2; HW 14.3; MXHW 18.3; IND 5.4; AGD 30.9; TRL 52.3; TAL 72.8; VL 6.5; FLL 20.5; HLL 21.7; LJL 13.4; SL 6.6; IOD 7.6; UEW 2.5; UEL 4.4; OL 3.8; BTAW 7.8; MTAW 2.8; MXTAH 9.2; MTAH 7.9; ON 3.8; ICD 9.8; CW 12.3; NSD 2.3; $\mathrm{FL}_{\mathrm{I}} 2.0 ; \mathrm{FL}_{\mathrm{II}} 3.7$; $\mathrm{FL}_{\text {III }}$ 3.7; $\mathrm{FL}_{\mathrm{IV}} 2.3 ; \mathrm{TL}_{\mathrm{I}} 2.0 ; \mathrm{TL}_{\text {II }} 3.5 ; \mathrm{TL}_{\text {III }}$ 5.3; $\mathrm{TL}_{\mathrm{IV}}$ 5.3; $\mathrm{TL}_{\mathrm{V}}$ 5.3.

Variation. All males were generally similar in morphology and coloration and agreed with the holotype description in body proportions and coloration; variation of morphometric characters within the type series is shown in Table 3. Variation of the dorsal coloration in four male paratypes in life is shown in Fig. 4. Males ZMMU A-7578 and ZMMU A-7582 had slightly lighter brownish coloration of dorsum. The number of rib nodules varies from 10 to 14 (mean 12.1 $\pm 1.1 ; n=10$ ). Male ZMMU A-7580 had notably shorter tail than other type specimens (Table 3; Fig. 4) likely due to regeneration of tail tip after damage; this specimen also had short regenerated fingers on left forelimb and toes on right hindlimb. In male ZMMU A-7578 second finger on left forelimb is regenerated and is notably curved (Fig. 4). Male ZMMU A-7581 lacked forearm on the right forelimb, also due to some sort of damage. The reasons behind the high proportion of regenerated limbs appendages in the new species is not clear, it may be related to possible combat between the males during the courtship season, or may also result from predators.

Etymology. The specific name "thaiorum" is a Latin adjective in the genitive plural (masculine gender), derived from the name of the Thai people who inhabit the montane areas of northern Vietnam and adjacent territories, including the type locality of the new species. The people of Muong Dan Village pointed out to us the breeding habitat of the new species. We suggest the following common names: Thai Crocodile Newt (English), Cá cóc thái (Vietnamese), Taiskiy krokodilovyi triton (Russian).

Comparisons. According to the results of our phylogenetic analyses, Tylototriton thaiorum sp. nov. falls into clade 1 of the subgenus Yaotriton and morphological comparisons with members of this group appear to be the most pertinent. The differences of the new species from all other congeners of the subgenus Yaotriton are summarized in Table 4. The new species can be easily distinguished from all members of the subgenus Tylototriton (clades 45 in Fig. 2) and $T$. panhai by dark body coloration except for bright orange palms and soles, vent region, and ventral ridge of tail ( $v s$ light color markings present on parotoids, lips, vertebral ridge, rib nodules, limbs, and ventral tail ridge). The new species can be distinguished from $T$. anhuiensis, $T$. broadoridgus, $T$. dabienicus, T. lizhenchangi, T. wenxianensis and $T$. vietnamensis by having prominent bony ridges on head, denser granules on body, large and small warts among the rib nodules, and distinct interspaces between rib nodules ( $v s$ less developed ridges on head, smoother skin, slightly flattened to nearly indistinct rib nodules). The new species further differs from $T$. vietnamensis by having dark brown coloration of dorsum ( $v s$ gray to tan body coloration). The new species can be distinguished from $T$. ziegleri by smaller rib nodules ( $v s$ enlarged knob-like rib nodules) and vertebral ridge wide, not segmented and rough ( $v s$ distinctly tubercular). Tylototriton thaiorum sp. nov. can be distinguished from $T$. asperrimus and $T$. 


\section{Plate 01}

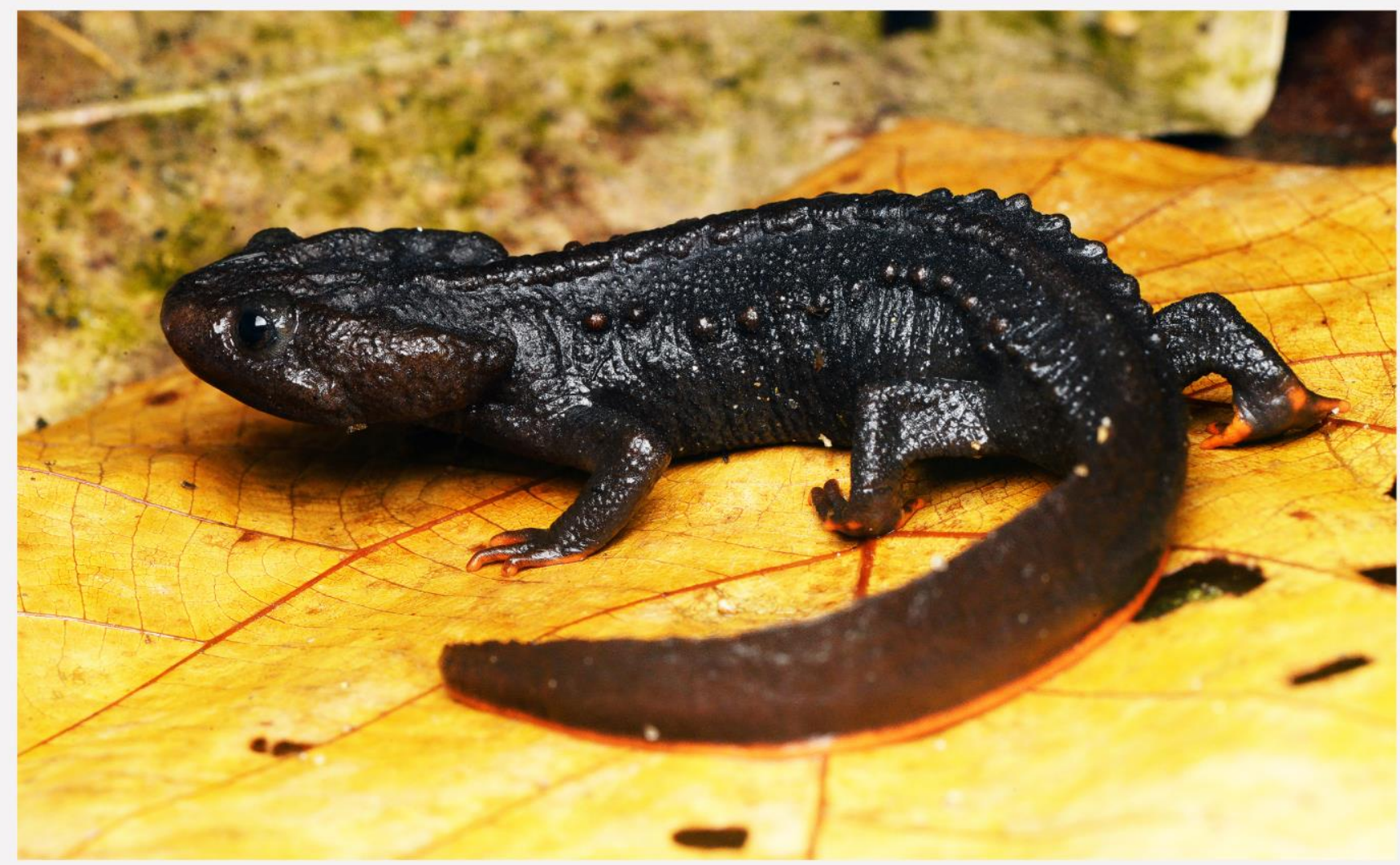

Figure 3. Holotype of Tylototriton thaiorum sp. nov. male in life (collected, ZMMU A-7577). Photo C: Nikolay A. Poyarkov.

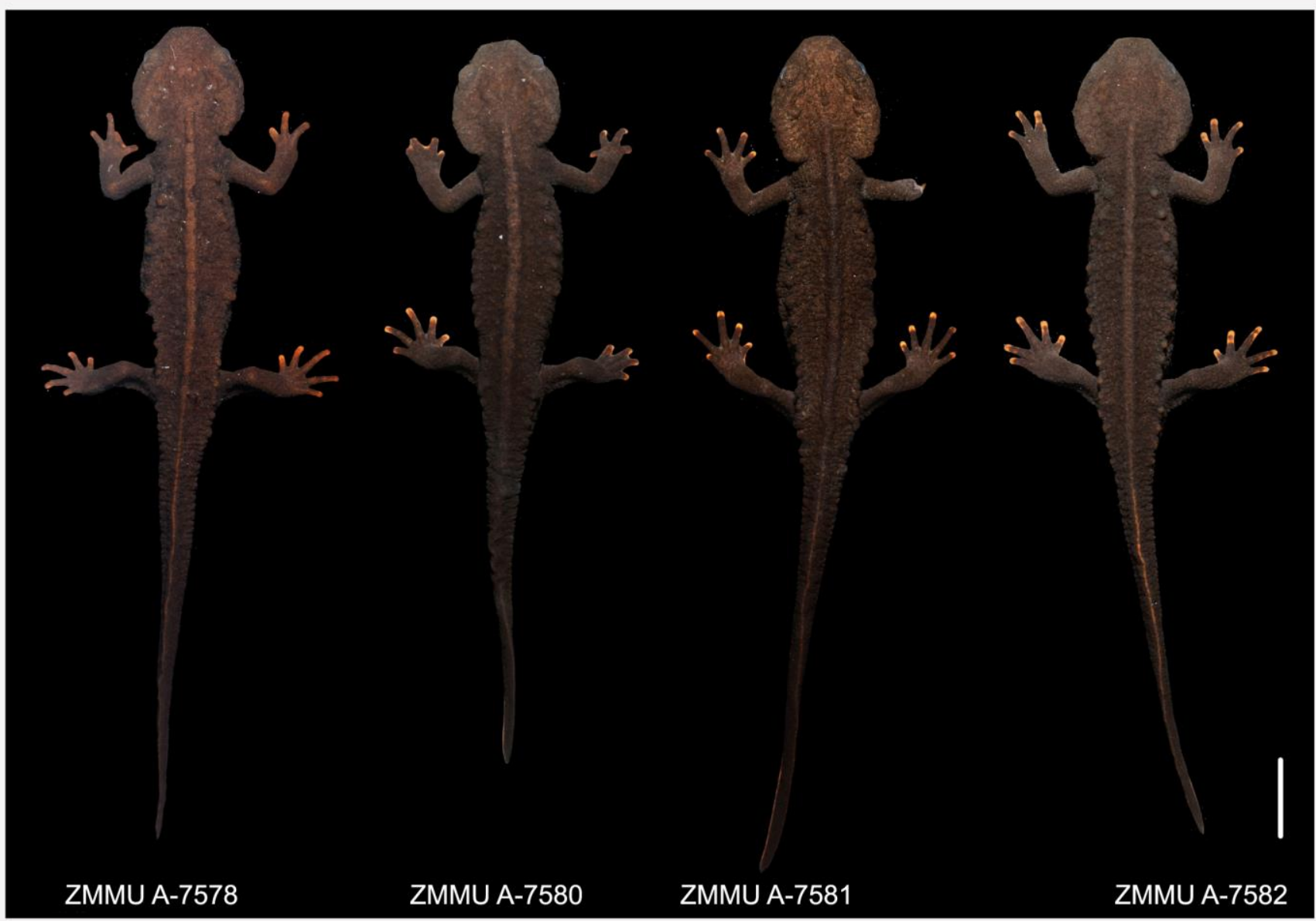

Figure 4. Variation of dorsal coloration in four adult male paratypes (ZMMU A-7578, 7580-82) of Tylototriton thaiorum sp. nov. (scale: $10 \mathrm{~mm}$ ). Photos C): Nikolay A. Poyarkov. 


\section{Plate 02}

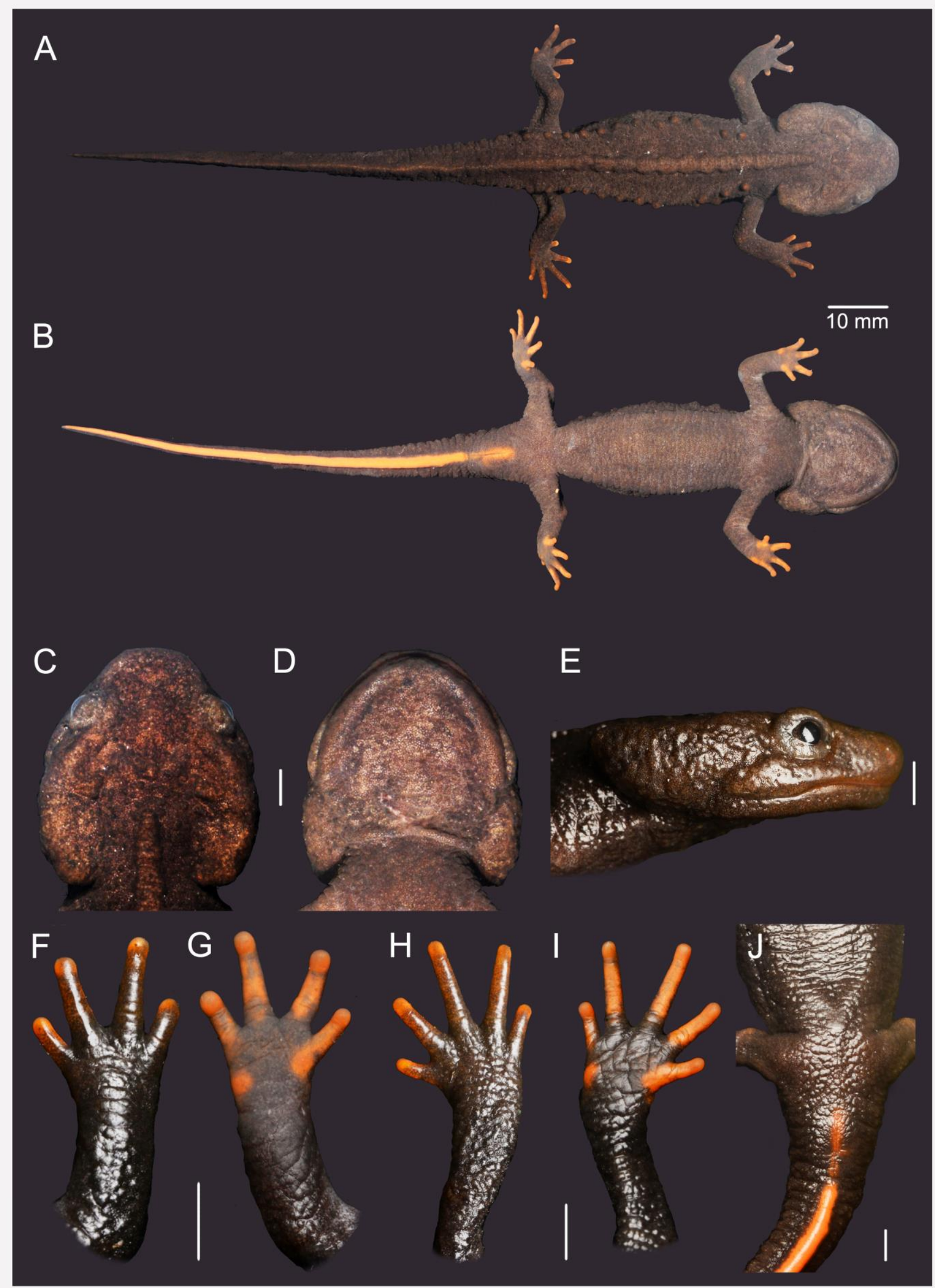

Figure 5. Holotype of Tylototriton thaiorum sp. nov. male in life (collected, ZMMU A-7577): body (A) dorsal and (B) ventral views; head (C) dorsal, (D) ventral, and (E) lateral views; right hand (F) dorsal and (G) ventral views; right foot (H) dorsal and (I) ventral views; (J) cloacal area (scale: $3 \mathrm{~mm}$ ) Photos $\mathbb{C}$ : Nikolay A. Poyarkov. 
hainanensis by lacking orange color markings on posterior parotoids ( $v s$ present), and light brown coloration on the rib nodules ( $v S$ uniformly black). The new species can be distinguished from $T$. liuyangensis by having smaller body size in males (male SVL 59.3-68.6 $\mathrm{mm}$ vs $64.2-82.0 \mathrm{~mm}$ ), by having light brown coloration on the rib nodules ( $v s$ uniformly black), and by ventral granules arranged in transverse striations ( $v s$ no transverse striations on belly). The new species is distinguished from T. maolanensis by having smaller body size in males (male SVL 59.3-68.6 mm vs 76.8-85.2 $\mathrm{mm}$ ), by snout obtusely rounded in dorsal view (vs truncate), by comparatively narrower vertebral ridge ( $v s$ very wide vertebral ridge). The new species can be diagnosed from $T$. pasmansi and $T$. sparreboomi by having snout obtusely rounded in dorsal view ( $v s$ truncate), and by having light brown coloration on the rib nodules ( $v s$ uniformly black). In general morphology, Tylototriton thaiorum sp. nov. superficially most closely resembles its sister species $T$. notialis, however the new species can be readily distinguished from the latter by having the following suite of morphological characters: (1) absence of orange coloration on posterior end of parotid ( $v s$ presence); (2) lightbrown coloration on rib nodules ( $v s$ bright orange), (3) a comparatively broader head in males: HW/HL 0.92-0.96 (mean 0.94 \pm 0.02 , $n=6$ ) vs 0.81 (mean $0.81 \pm 0.00, n=2$; data from Stuart et al. 2010); (4) obtusely rounded snout in dorsal view ( $v s$ truncated, slightly angular snout); (5) relatively shorter forelimb length in males: FLL/SVL 0.31-0.34 (mean 0.32 \pm 0.01 , $n=6$ ) vs $0.39-0.41$ (mean $0.40 \pm 0.01, n=2$; data from Stuart et al. 2010); (6) relatively shorter forelimb length in males: HLL/SVL 0.33-0.36 (mean $0.35 \pm 0.01, n=6$ ) vs $0.38-0.40$ (mean $0.39 \pm 0.01, n=2$; data from Stuart et al. 2010); (7) slightly smaller size of rib nodules intermittent with numerous smaller warts and arranged in irregular series, rib nodules notably vary in size and shape ( $v s$ generally larger rounded rib nodules of approximately uniform size and shape, arranged in regular dorsolateral series lacking intermittent smaller warts in gaps between them); (8) lower number of rib nodules: $10-14$, mean $12.1 \pm 1.1, n=12$ (vs 14-16, mean $15.2 \pm 0.8, n=6$; data from figure 3 in Stuart et al. 2010, and figure 1 in Nishikawa et al. 2020).

Distribution and biogeography. To date, Tylototriton thaiorum sp. nov. is known only from a restricted area in montane forests of $\mathrm{Pu}$
Hoat Nature Reserve, Nghe An Province, northern part of Central Vietnam (Fig. 1) at elevations from 700 to $1000 \mathrm{~m}$ a.s.l. This area is located ca. $220 \mathrm{~km}$ air distance northwards from the type locality of $T$. notialis in Khammouan Province of Laos. The Pu Hoat Mountain Range is located on the northeastern edge of Xiangkhouang Plateau; it separates the Nghe An Province of Vietnam from the Houaphan Province of Laos. The locality of the new species is ca. $4 \mathrm{~km}$ from the national border of Vietnam and Laos, thus the occurrence of Tylototriton thaiorum sp. nov. is also anticipated on the north-western slopes of $\mathrm{Pu}$ Hoat Mountain Range in the adjacent parts of Houaphan Province of Laos.

Natural history. Our knowledge of the biology of Tylototriton thaiorum sp. nov. is scarce. Adult male newts were encountered at night after $1900 \mathrm{~h}$ in the water in ephemeral swamps in forest clearings. Surrounding areas were covered by dominant evergreen montane subtropical forest and secondary bamboo forest. Adult males were moving slowly along the muddy bottom in clear water $20-100 \mathrm{~cm}$ deep; the temperatures at the time of collection were air $25^{\circ} \mathrm{C}$, and water $22^{\circ} \mathrm{C}$. Females and larvae of the new species were not observed. We assume that the new species, similar to other members of the subgenus Yaotriton, is basically terrestrial and cannot be found in the water except during the breeding season. Other syntopic species of amphibians recorded at the type locality include: Leptobrachium masatakasatoi Matsui, 2013, Sylvirana maosonensis (Bourret, 1937), Rhacophorus orlovi Ziegler \& Köhler, 2001, Polypedates cf. mutus (Smith, 1940), Limnonectes limborgi (Sclater, 1892) and Microhyla pulchra (Hallowell, 1861).

Conservation status. Tylototriton thaiorum sp. nov. is to date known only from a single location in $\mathrm{Pu}$ Hoat Nature Reserve, Nghe An Province of Vietnam, and is expected to be recorded in the adjacent parts of the Houphan Province of Laos. Though the actual extent of distribution of the new species remains unknown, it is likely a range-restricted species, restricted to the $\mathrm{Pu}$ Hoat Mountain Range. Habitat loss due to intensifying agriculture and forest logging and collection (locally) for traditional medicinal use (for abdominal pain and some parasitic infections) are among the major threats. We thus suggest Tylototriton thaiorum sp. nov. to be listed as Endangered (EN) [IUCN Red List criteria B1ab(iii)]. 


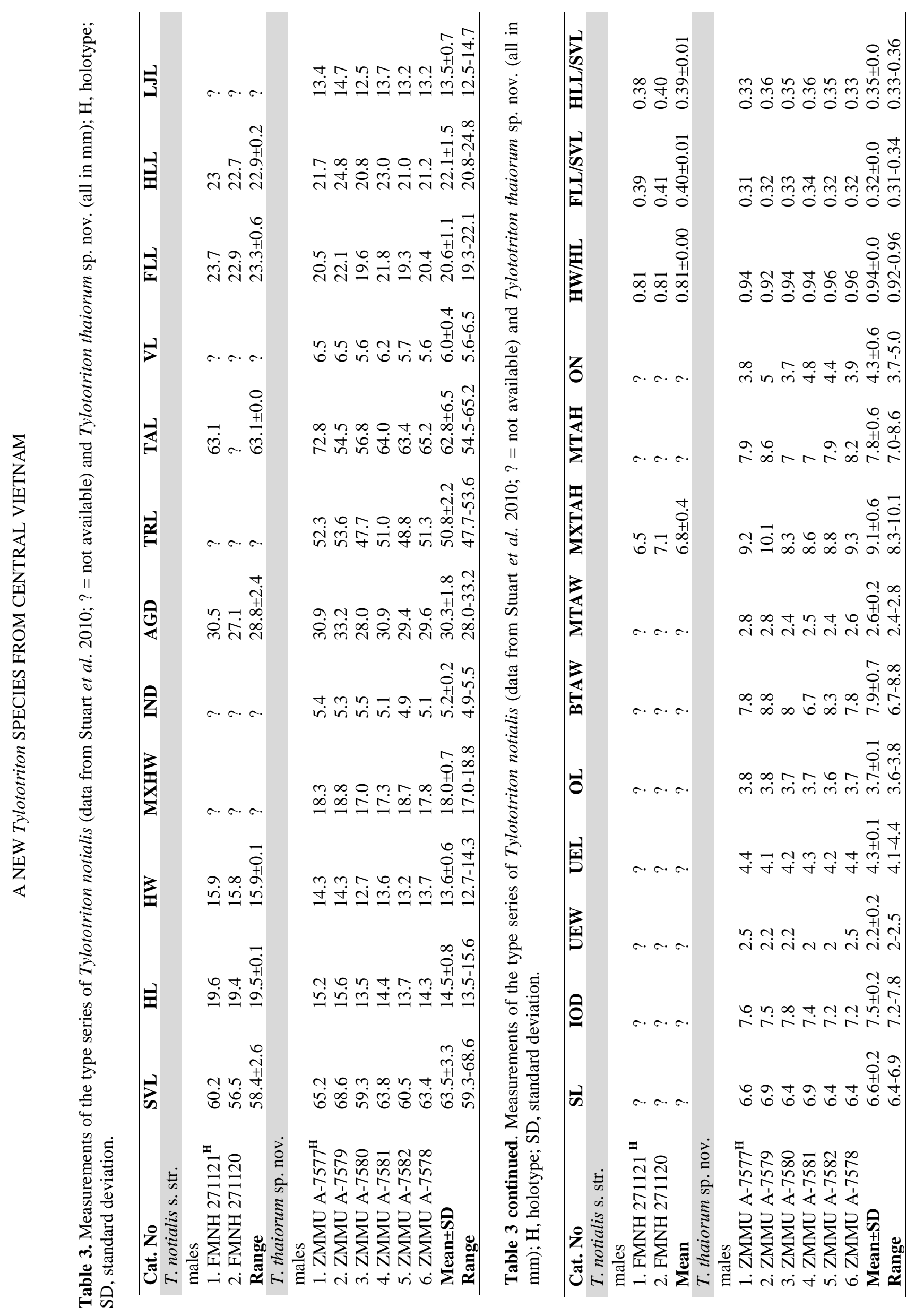




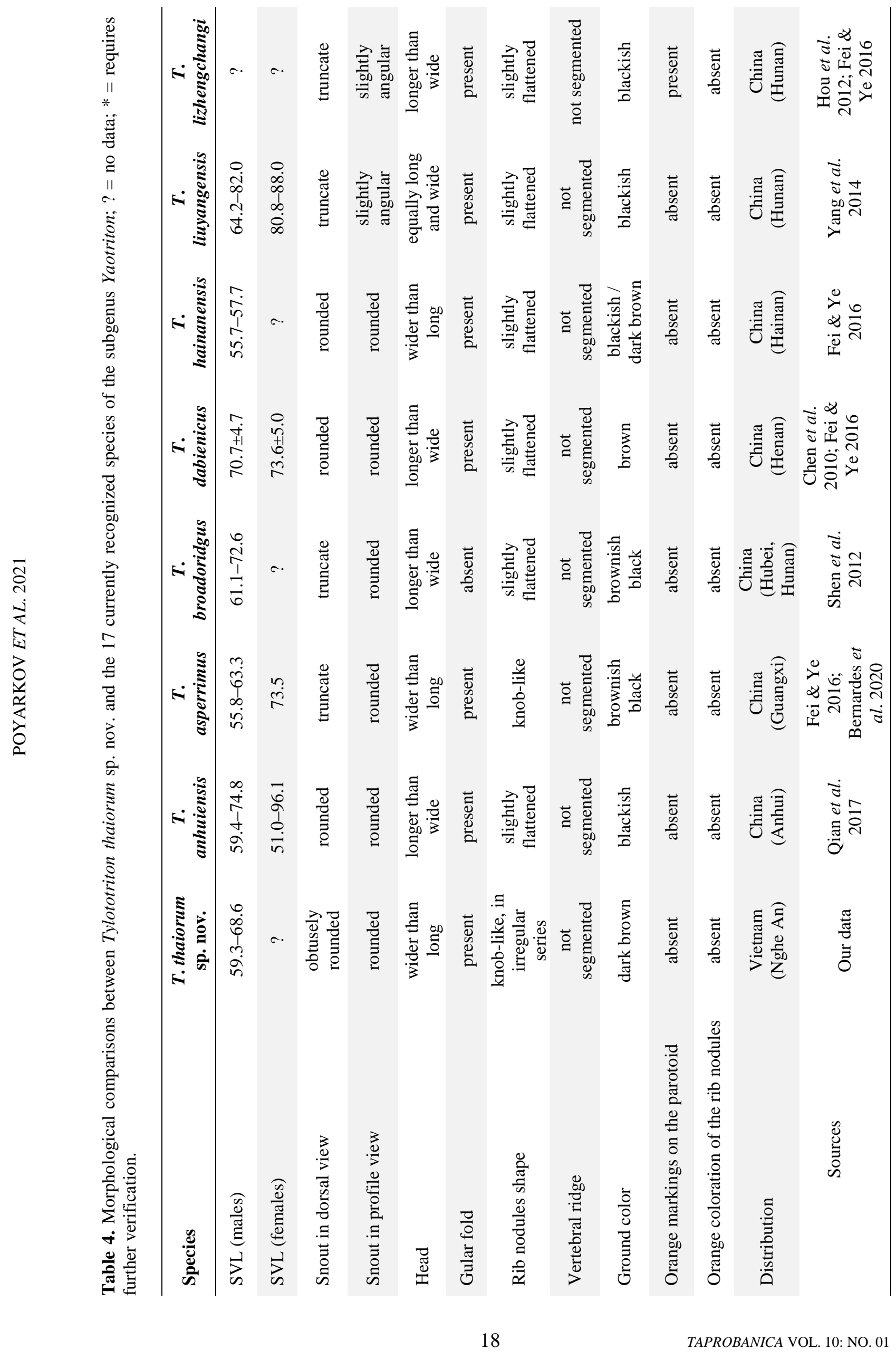




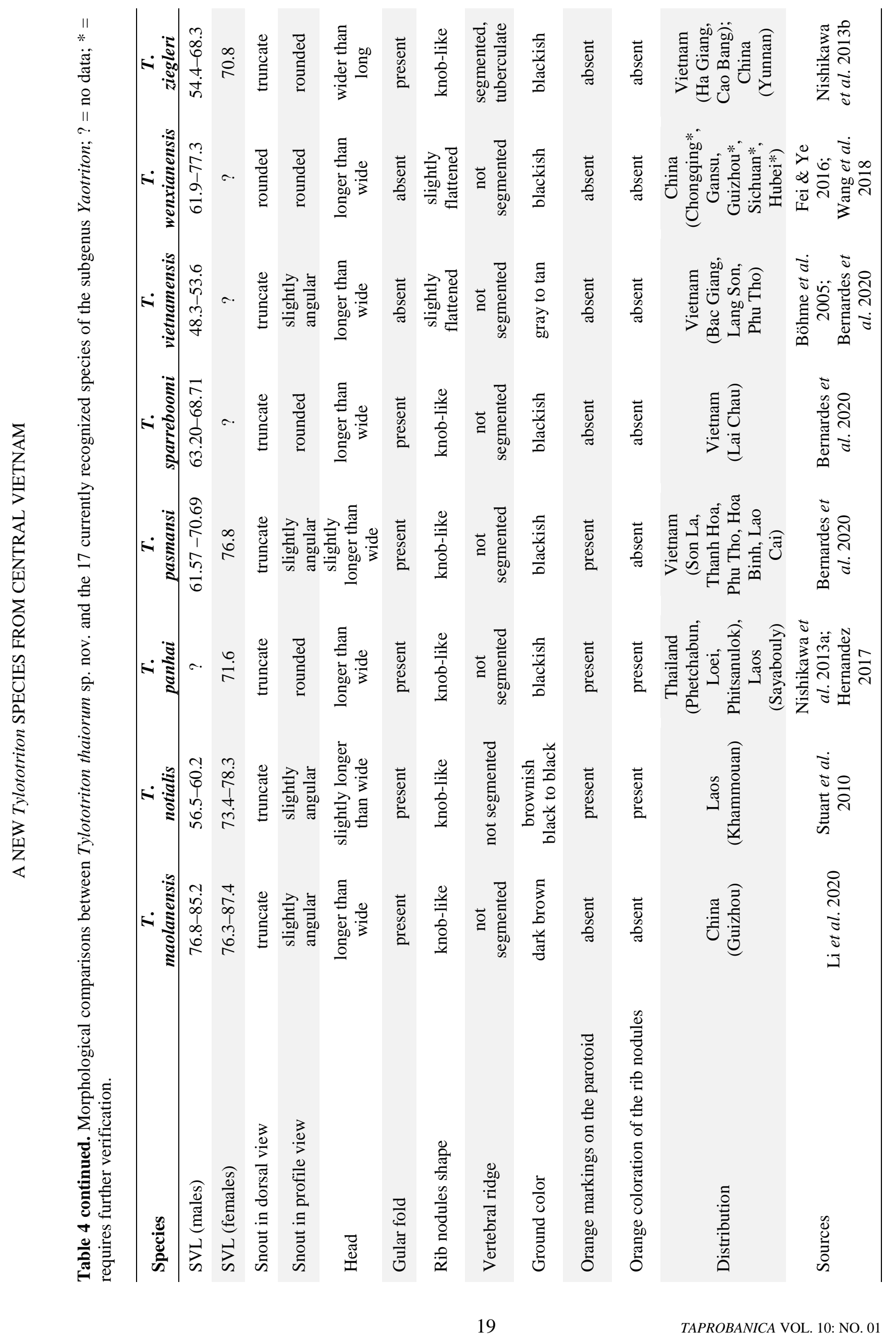




\section{Discussion}

The range of Tylototriton thaiorum sp. nov. seems to be restricted to the $\mathrm{Pu}$ Hoat Mountain Range located at the southernmost edge of the Northern Indochina subtropical forests ecoregion according to the terrestrial ecoregion classification of Olson et al. (2001). Its sister species $T$. notialis is located in the heart of the northern part of the Truong Son Mountain Range, which belongs to the Northern Annamites rain forests ecoregion (Olson et al. 2001). These areas differ markedly in the amount of annual rainfall, climate types, and vegetation (e.g. Pham \& Phan 1993, Nguyen et al. 2000, Averyanov et al. 2003), and correspond to different biogeographic subregions of Indochina for herpetofauna (Bain \& Hurley 2011, Poyarkov et al. 2021 in press). These two ecoregions are separated by the valley of the Ca River, which has been shown to be an important biogeographic barrier for vascular plants (Averyanov et al. 2003), insectivorous mammals (Abramov \& Tran 2017), and amphibians (Bain \& Hurley 2011, Poyarkov et al. 2021 in press). Since the Ca River is the largest river in the northern part of Central Vietnam, its valley may serve as a physical barrier restricting gene flow between Tylototriton populations. Further studies, including intensive field surveys in the Annamite part of Nghe An and Ha Tinh provinces of Vietnam, are required to clarify the role of the $\mathrm{Ca}$ River valley in divergence between $T$. thaiorum and $T$. notialis.

Our phylogenetic analysis confirmed the earlier results of Wang et al. (2018), Than Zaw et al. (2019), and Li et al. (2020), which suggest that our knowledge on the actual taxonomic diversity of Tylototriton still remains incomplete. Based on our mtDNA genealogy, at least two lineages of the $T$. wenxianensis species group (Tylototriton sp. 1 from Guizhou Province, China, and Tylototriton sp. 2 from Hubei Province, China) and one lineage of the $T$. asperrimus species group (Tylototriton sp. 3 from Guangdong Province, China) likely represent undescribed new species. Further integrative taxonomic studies are required to clarify the taxonomic status of these lineages. Moreover, our analysis demonstrated that the level of genetic differentiation between the two subspecies of $T$. pasmansi from Vietnam ( $p=$ $4.2 \%$ for ND2 gene) actually corresponds to the species level of divergence in Tylototriton. Bernardes et al. (2020) have cautiously described T.p. pasmansi and T. pasmansi obsti as subspecies, largely due to the limited sample size they could examine as well as due to the lack of clear morphological differences between these lineages. We call for additional field surveys and genetic and morphological studies which are required for a comprehensive taxonomic evaluation of these taxa.

Our description of $T$. thaiorum brings the total number of recognized Tylototriton species to 32 . The previous record of $T$. notialis from Vietnam is now revised as $T$. thaiorum in this study; therefore the total number of Tylototriton species (7) known from Vietnam remains unchanged. The occurrence of $T$. notialis sensu stricto in Vietnam is nevertheless expected in the parts of the Northern Annamites adjacent to the Khammouan Province of Laos; further surveys in montane areas of Quang Binh and $\mathrm{Ha}$ Tinh provinces of Vietnam may lead to discovery of new populations of this species. China with 17 species and Vietnam with seven species, currently host the largest proportion of the Tylototriton newt diversity; four of the seven Tylototriton species recorded in Vietnam are endemic to the country. The Vietnamese populations of Tylototriton are under constantly growing threat from many pressures such as habitat loss, water pollution, and poaching for the pet trade (Rowley et al. 2016, Bernardes et al. 2017, 2020, Grismer et al. 2018). Hernandez et al. (2018) suggested that niche conservatism along with niche differentiation shape the patterns of macro-ecological niche differentiation in Tylototriton. Most species are restricted to cooler and more humid highelevation forested habitats, while lowland and open areas are unsuitable for the Tylototriton newts (Hernandez et al. 2018). Hence, the unsuitable micro-climatic conditions in the lowland regions of Indochina might limit dispersal and gene flow in Tylototriton. Further intensive fieldwork efforts in different isolated mountain massifs of northern Indochina, including central and northern Vietnam and the adjacent parts of Laos are required to obtain a more comprehensive understanding of Tylototriton diversity and distribution in the region.

\section{Acknowledgements}

This study was funded by the Russian Science Foundation (RSF grant No. 19-14-00050), and the fieldwork was organized and funded by the Joint Russian-Vietnamese Tropical and 
Technological Centre (JRVTTC) conducted under permission of the Forest Protection Department of the Peoples' Committee of Nghe An Province and the Department of Forestry, Ministry of Agriculture and Rural Development of Vietnam (permit nos. 1700/UBN.VX, 22.03.2018; 2089/UBN.VX，03.04.2019). We thank Andrey N. Kuznetsov, Hoi D. Nguyen (JRVTTC), Leonid P. Korzoun (MSU), and Thai $\mathrm{V}$. Nguyen (SVW) for the support and organization of fieldwork; Chung and the community of Muong Dan Village (Que Phong District, Nghe An Province) for assistance during the fieldwork; Vladislav A. Gorin for assistance in the lab. We thank L. Lee Grismer (La Sierra University, California) and Yang Jianhuan (Kadoorie Conservation China Dept., Hong Kong) for reviewing the manuscript.

\section{Literature cited}

Abramov, A.V. and T.Q. Tran (2017). Zoogeography of insectivorous mammals of Vietnam. Tạp chí khoa hoc và công nghệ nhiệt đói / Journal of Tropical Science and Technology, Hanoi, 14 (11): 49-58.

Averyanov, L.V., L.K. Phan, H.T. Nguyen, and D.K. Harder (2003). Phytogeographic review of Vietnam and adjacent areas of Eastern Indochina. Komarovia, 3: 1-83.

Bain, R.H. and M.M. Hurley (2011). A biogeographic synthesis of the amphibians and reptiles of Indochina. Bulletin of the American Museum of Natural History, 360: 1-138.

Bernardes, M., M.D. Le, T.Q. Nguyen, C.T. Pham, and A.V. Pham AV et al. (2020). Integrative taxonomy reveals three new taxa within the Tylototriton asperrimus complex (Caudata, Salamandridae) from Vietnam. ZooKeys, 935: 121-164.

Bui M. Q, M. A. T. Nguyen, and von A. Haeseler (2013). Ultrafast approximation for phylogenetic bootstrap. Molecular Biology \& Evolution, 30: 1188-1195.

Böhme, W., T. Schöttler, T.Q. Nguyen, and J. Köhler (2005). A new species of salamander, genus Tylototriton (Urodela: Salamandridae) from northern Vietnam. Salamandra, 41 (4): 215-220.

Chen, X.H., X.W. Wang, and J. Tao. (2010). A new subspecies of genus Tylototriton from China (Cudata, Salamandridae). Acta Zootaxonomica Sinica, 35 (3): 666-670. (in Chinese).

Fei, L. and C-Y. Ye (2016). Amphibians of China, Volume 1. [place unknown]: Beijing, China: Chengdu Institute of Biology, Chinese Academy of Sciences. Science Press.
Frost, D.R. (2021). Amphibian Species of the World: An Online Reference. Version 6.1. <www.amphibiansoftheworld.amnh.org/>. Accessed on 20 March 2021.

Grismer, L.L., P.L. Wood, Jr., E.S.H. Quah, M.K. Thura et al. (2018). A new species of Crocodile Newt Tylototriton (Caudata: Salamandridae) from Shan State, Myanmar (Burma). Zootaxa, 4500 (4): 442-573.

Hall, T.A. (1999). BioEdit: a user-friendly biological sequence alignment editor and analysis program for Windows 95/98/NT. Nucleic Acids Symposium Series, 41: 95-98.

Hedges, S.B. (1994). Molecular evidence for the origin of birds. Proceedingsof the National Academy of Sciences of the United States of America, 91 (7): 2621-2624.

Hernandez, A. (2016). Crocodile newts-the Primitive Salamandridae of Asia (Genera Echinotriton and Tylototriton). Edition Chimaira, Frankfurt am Main, Germany, 416pp.

Hou, M., P. Li, and S.Q. Lü (2012). Morphological research development of genus Tylototriton and primary confirmation of the status of four cryptic populations. Journal of Huangshan University, 14 (3): 61-65 (in Chinese).

Hoang, D.T., O. Chernomor, A. von Haeseler, M.Q. Bui, and V.S. Le (2018). UFBoot2: Improving the ultrafast bootstrap approximation. Molecular Biology \& Evolution, 35: 518-522.

Huelsenbeck, J.P. and F. Ronquist (2001). MRBAYES: Bayesian inference of phylogenetic trees. Bioinformatics, 17 (8): 754-755.

IUCN Standards and Petitions Committee (2019). Guidelines for using the IUCN Red List categories and criteria version 14. Geneva, Switzerland. Prepared by the Standards and Petitions Committee: 113 pp.

Jiang, Y., Z. Wei, F. Han, Q. Ni., and Y. Yao et al. (2017). The complete mitogenome sequence of Tylototriton ziegleri (Amphibia: Caudata). Conservation Genetics Resources, 9 (3): 503506.

Katoh, K., K. Misawa, K. Kuma, and T. Miyata (2002). MAFFT: a novel method for rapid multiple sequence alignment based on fast Fourier transform. Nucleic Acids Research, 30 (14): 3059-3066.

Kumar, S., G. Stecher, and K. Tamura (2016). MEGA7: Molecular Evolutionary Genetics Analysis version 7.0 for bigger datasets. Molecular Biology \& Evolution, 33(7): 18701874. 
Le, D.T., T.T. Nguyen, K. Nishikawa, S.L.H. Nguyen, and A.V. Pham et al. (2015). A new species of Tylototriton Anderson, 1871 (Amphibia: Salamandridae) from Northern Indochina. Current Herpetology, 34 (1): 3850.

Li, S., G. Wei, Y. Cheng, B. Zhang, and B. Wang (2020). Description of a new species of the Asian Newt genus Tylototriton sensu lato (Amphibia: Urodela: Salamandridae) from southwest China. Asian Herpetological Research, 11: 282-296.

Nguyen, V.K., H.T. Nguyen, L.K. Phan, and H.T. Nguyen (2000). Bioclimatic diagrams of Vietnam [in Vietnamese] Vietnam National University Publishing House, Hanoi: 126pp.

Nishikawa, K., D. Sanamxay, T. Kurita, M. Hibino, and T. Jono et al. (2020). A new and highest record of Tylototriton notialis (Amphibia: Urodela: Salamandridae). Academia Journal of Biology, 42: 135-137.

Nishikawa, K., M. Matsui, and T.T. Nguyen (2013b). A new species of Tylototriton from northern Vietnam (Amphibia: Urodela: Salamandridae). Current Herpetology, 32 (1): 34-49.

Nishikawa, K., W. Khonsue, P. Pomchote, and M. Matsui (2013a). Two new species of Tylototriton from Thailand (Amphibia: Urodela: Salamandridae). Zootaxa, 3737 (3): 261-279.

Okamiya, H., H. Sugawara, M. Nagano, and N.A. Poyarkov (2018). An integrative taxonomic analysis reveals a new species of lotic Hynobius salamander from Japan. PeerJ, 6: e5084.

Olson, D.M., E. Dinerstein, E.D. Wikramanayake, N.D. Burgess et al. (2001). Terrestrial Ecoregions of the World: A new map of life on Earth. A new global map of terrestrial ecoregions provides an innovative tool for conserving biodiversity. BioScience, 51 (11): 933-938.

Pham, T.N. and D.T. Phan (1993). The climate of Vietnam. Edition 2 [in Vietnamese]. Nhà xuất bản Khoa học và Kỹ thuật / Publishing house for Science and Technology, Hanoi: 312pp.

Phimmachak, S., A. Aowphol, and B.L, Stuart (2015). Morphological and molecular variation in Tylototriton (Caudata: Salamandridae) in Laos, with description of a new species. Zootaxa, 4006 (2): 285-310.

Posada, D. and K.A. Crandall (1998). MODELTEST: testing the model of DNA substitution. Bioinformatics, 14 (9): 817-818.
Poyarkov, N.A., J. Che, M.S. Min, M. Kuro-o, and F. Yan et al. (2012). Review of the systematics, morphology and distribution of Asian Clawed Salamanders, genus Onychodactylus (Amphibia, Caudata: Hynobiidae), with the description of four new species. Zootaxa, 3465 (1): 1-106.

Poyarkov, N.A., T.V. Nguyen, E.S. Popov, P. Geissler, P. Pawangkhanant et al. (2021). Recent progress in taxonomic studies, biogeographic analysis and revised checklist of amphibians in Indochina. Russian Journal of Herpetology, in press.

Qian, L.-F., X.-N. Sun, J.-Q. Li, W.-B. Guo, and T. Pan et al. (2017). A new species of the genus Tylototriton (Amphibia: Urodela: Salamandridae) from the southern Dabie Mountains in Anhui Province. Asian Herpetological Research, 8: 151-164.

Rambaut, A., M. Suchard, W. Xie, and A. Drummond (2014). Tracer v. 1.6. Institute of Evolutionary Biology, University of Edinburgh. Available at: http://tree.bio. ed.ac.uk/software/tracer/.

Ronquist, F., and Huelsenbeck (2003). MRBAYES 3: Bayesian phylogenetic inference under mixed models. Bioinformatics, 19 (12): 15721574.

Shen, Y.H., J. Jiang, and X. Mo. (2012). A new species of the genus Tylototriton (Amphibia, Salamandridae) from Hunan, China. Asian Herpetological Research, 3 (1): 21-30.

Stuart, B.L., S. Phimmachak, N. Sivongxay, and W.G. Robichaud (2010). A new species in the Tylototriton asperrimus group (Caudata: Salamandridae) from central Laos. Zootaxa, 2650 (1): 19-32.

Than Zaw, P. Lay, P. Pawangkhanant, V.A. Gorin, and N.A. Poyarkov (2019). A new species of Crocodile newt, genus Tylototriton (Amphibia, Caudata, Salamandridae) from the mountains of Kachin state, northern Myanmar. Zoological Research, 40 (3): 151-174.

Wang, B., K. Nishikawa, M. Matsui, T.Q. Nguyen, and F. Xie et al. (2018). Phylogenetic surveys on the newt genus Tylototriton sensu lato (Salamandridae, Caudata) reveal cryptic diversity and novel diversification promoted by historical climatic shifts. PeerJ, 6: e4384.

Yang, D., J. Jiang, Y. Shen, and D. Fei (2014). A new species of the genus Tylototriton (Urodela: Sala mandridae) from northeastern Hunan Province, China. Asian Herpetological Research, 5 (1): 1-11. 